\title{
The impact of extreme genu varum and genu valgum on postoperative outcomes using mini-subvastus approach in TKA
}

\author{
Bogdan Ştefan Crețu* **, Călin Dragosloveanu *, Dragoş Cotor *, \\ Şerban Dragosloveanu ***, Cristian loan Stoica* ** \\ * "Foişor" Orthopaedics-Traumatology and OsteoarticularTB Hospital, Bucharest, Romania \\ ** "Carol Davila" University of Medicine and Pharmacy, Bucharest, Romania
}

Correspondence to: Bogdan Ştefan Crețu, MD, PhD, "Foişor" Orthopaedics-Traumatology and Osteoarticular TB Hospital, Bucharest, 35-37 Ferdinand I Blvd., Bucharest, Romania, Mobile phone: 0040741127187, E-mail: jfrbogdan@yahoo.com

\begin{abstract}
Increasing interest in using minimally invasive approaches in TKA has led to the question: how much deformity is accepted for using MIS in TKA? A single surgeon performed 87 consecutive TKAs with mini-subvastus approach, using unconstrained prosthesis in 84 knees and constrained prosthesis in 3 knees. We conducted a prospective study in which patients were divided into two groups according to preoperative tibiofemoral axes (TFM), one group with $160^{\circ}<\mathrm{TFM}<195^{\circ}$ and the second group with $160^{\circ} \geq \operatorname{TFM} \geq 195^{\circ}$. Clinical and radiographic outcomes were compared. Postoperative ROM and knee score were improved in both groups, with similar results $(p<0.01)$. Postoperative radiographic analyses showed that TFM was improved in both groups $(p<0.01)$ with the coronal alignment inferior in $160^{\circ} \geq T F M \geq 195^{\circ}$ group than the $160^{\circ}<\mathrm{TFM}<195^{\circ}$ group. The results of this study suggest that mini-subvastus approach is a proper technique to use in primary TKA in patients with a TFM angle less than $160^{\circ}$ and more than $195^{\circ}$ with similar results with TFM angle between $160^{\circ}$ and $195^{\circ}$, and for the use of constrained prosthesis designs with promising results. Preoperative TFM angle less than $160^{\circ}$ and greater than $195^{\circ}$ increases the risk of component malposition in coronal plane.
\end{abstract}

Keywords: minimally invasive, total knee arthroplasty, implant positioning, tibiofemoral axe, constrained prosthesis

\section{Introduction}

Minimally invasive surgery (MIS) in total knee arthroplasty (TKA) began in the late 1990's and has been evolving since then due to its benefits of reduced pain, blood loss, return of function and hospitalization time, and to improve functional recovery [1-4]. Increasing interest using minimally invasive approaches in TKA has led to the question whether MIS has a role in primary TKA for severe deformities using unconstrained and constrained prosthesis. Minimally invasive TKA has evolved rapidly thanks to new instrumentation and technique. The definition of MIS includes a smaller skin incision, no eversion of the patella, minimally 
disruption of the patellar tendon, minimally disruption of the suprapatellar pouch, no dislocation of the tibiofemoral joint. The main approaches in MIS TKA are quadriceps sparing, limited parapatellar, limited subvastus, and limited midvastus [4]. The limited parapatellar technique uses a smaller parapatellar arthrotomy than the traditional parapatellar arthrotomy $[5,6]$. The limited midvastus uses an arthrotomy that extends $2 \mathrm{~cm}$ or more into the vastus medialis muscle [7]. The quadriceps sparing method uses a more medial incision avoiding a quadriceps arthrotomy [1,8-10]. The limited subvastus is an evolution of Hoffman technique which uses an anterior approach and a quadriceps arthrotomy [5,11]. Limited subvastus approach provides good exposure, preserves the quadriceps insertions on the patella, and does not require patellar eversion [12]. The selection criteria for using MIS techniques are patient weight, body mass index, age, previous surgery, knee deformity, preoperative range of motion $[1,3,5,6,9,10,13]$. The actual experience with MIS TKA defines the following indications: less than $15^{\circ}$ of varus, $20^{\circ}$ of valgus, or a flexion contracture less than $10^{\circ}$, range of motion less than $90^{\circ}$ [14]. Not all patients are proper candidates for MIS TKA, like obese or large-muscled male patients, patients with a wide femur or with short patellar tendon. There is evidence that the use of MIS TKA reduces postoperative pain and facilitates faster recovery after surgery [15]. However, the deficient exposure can cause component malalignment and early failure after MIS TKA [16]. The main concerns are vascular injury, patellar tendon injury, condylar fracture, wound dehiscence, and necrosis [17]. According to William C. Schroer et al., the restrictive indications for using MIS techniques in TKA raise the concern that positive clinical outcomes are due to patient rigorous selection rather than surgical technique [7]. The plasticity of the MIS techniques allows surgeons to adapt and modify the procedure thereby expanding its indications such as the limited subvastus approach, which can be used in the majority of primary total knees arthroplasty. Our hypothesis is that limited subvastus is an adequate approach to be used in severe genu varum and severe genu valgum with the TFM angle less than $160^{\circ}$ and greater than $195^{\circ}$ and with the constrained designs prosthesis.

\section{Materials and methods}

The study included a number of 87 -knee osteoarthritis that underwent TKA using minisubvastus surgical technique, in 84 patients. The same surgeon in the same clinic performed all the surgeries between January 2014 and January 2017. The patient records were viewed prospectively. The mini-subvastus technique used in our study complies with the definition involving a smaller skin incision, no eversion of the patella, minimally disruption of the patellar tendon, minimally disruption of the suprapatellar pouch. Our surgical goal was to restore mechanical alignment (mechanical alignment of $\left.\circ^{\circ}\right)$, joint line, balanced ligaments (correct flexion and extension gaps) and maintain normal $\mathrm{Q}$ angle (ensures proper patellar femoral tacking). The surgeon used a posterior stabilized prosthesis, constrained non-hinged prosthesis, and constrained hinged prosthesis. The inclusion criteria consisted of loss of range of motion and important pain due to osteoarthritis, rheumatoid arthritis, without taking into consideration the degree of varus and valgus deformity measured with TFM (tibiofemoral mechanical axis), presence of flexion contracture, or presence of genu recurvatum. The exclusion criteria consisted of previous surgery of the knee requiring removal of metal implants and TKA revision. Patients were divided into two groups according to preoperative TFM: $160^{\circ}<\mathrm{TFM}<195^{\circ}$ group and the other group with $160^{\circ} \geq \operatorname{TFM} \geq 195^{\circ}$. In both groups, we measured preoperative and postoperative: ROM, The Knee Society Score (KSS) and the TFM angle. Postoperative radiographic assessment used was compliant 
with the guidelines of the Knee Society [18]. Postoperatively, we also analyzed the FCM angle (angle between femoral mechanical axes and transcondylar line from the femoral component), the Insall-Salvati ratio, and the patellar tilt.

\section{Radiographic evaluation}

Weight bearing anteroposterior (AP) and lateral radiographic views of the knee that evaluated joint space narrowing, collateral ligamentinsufficiencyviewedaslateralgapping, subluxations of the knee, bone defects. Long leg weight bearing AP and lateral radiographic views were performed to determine an accurate valgus cut angle when the patient had femoral or tibial deformity. Flexion lateral view and sunrise view radiographs were performed. Postoperative radiographs measurements included coronal TFM (angle between femoral and tibial mechanical axes on weight bearing long leg AP radiographs); valgus cutting angle (angle between anatomical ax of the femur AAF and mechanical ax of the femur $M F$, normal $5-7^{\circ}$ from AAF); coronal femoral component angle (a) (angle between femoral shaft and transcondylar line of the femoral component); coronal tibial component angle (b) (angle between mechanical axes of the tibia and tibial base plate); sagittal femoral component angle (c) (measures flexion of the femoral component, recommended $\mathrm{o}^{\circ}$ ); sagittal tibial component angle (d) (posterior slope of the tibial component, recommended $83^{\circ}$ ). We also measured the coronal femoral component angle to the femoral mechanical axes (FCM: angle between femoral mechanical axes and transcondylar line from the femoral component). Postoperative radiographic measurements included patellar tilt and the Insall-Salvati ratio for the patella, measured on the sunrise view and lateral X-ray with the knee in $30^{\circ}$ flexion. The Insall-Salvati ratio (patellar tendon length/ patellar length) is considered normal between 0.8 and 1.2. Patellar tilt angle measured between a line from the anterior limit of the femoral component and the line drawn down through the prosthesis-bone interface in the $60^{\circ}$ axial view [19].

\section{Surgical technique}

In all TKAs included in our study, we used a mini-subvastus approach that consisted in a skin incision centered midline from the superior pole of the patella to the tibial tubercle. The incision ranged from 7 to $12 \mathrm{~cm}$. An arthrotomy was created along the inferior border of vastus medialis obliquus (VMO) leaving a cuff of retinaculum for closure, which was continued along the medial border of the patella and the patellar tendon (Fig. 1). The synovial capsular reflexion underneath the VMO was released, and the patellar tendon fat pad was partially excised allowing the patella to sublux laterally without everting. A " $Z$ " retractor was placed under the VMO laterally, and the knee was flexed to $90^{\circ}$ degrees. We used the mobile window technique, which allowed access to all compartments of the knee in various positions of flexion and extension.

Femoral intramedullary and tibial extramedullary guides were used for the unconstrained prosthesis and intramedullary tibial and femoral guides were used for the constrained designs.

\section{Implants}

According to the types of prosthesis used, we had one group with an unconstrained design posterior stabilized prosthesis and one other group with constrained designs nonhinged and hinged prosthesis. We used the mini-subvastus approach for all TKAs. All tibial and femoral components were fixed using bone cement. For all the implants, we adhered to the technique stated by the manufacturer.

\section{Clinical pathway}

All the patients included in the study, who had undergone a TKA procedure, followed a standardized protocol. Each patient conducted standard radiographs, blood analyses, urine 
analyses. Each patient received identical thromboembolic prophylaxis using low weight molecular heparin. Preoperatively and intraoperatively, all the patients received prophylactic antibiotics (glycopeptides and 2 nd generation cephalosporins). Postoperatively, all the patients received the same physical therapy program. None of the patients used continuous passive motion machines. The anesthesia methods and postoperative pain medication was administered by the anesthesiologist. Discharge criteria included active-assisted knee range of motion $0^{\circ}-90^{\circ}$ in a seated position, the ability to ambulate, use stairs, independent with home exercise program.

\section{Outcome measurement}

Clinical and radiographic follow-up was done at discharge and at 3 months. Radiographic evaluation included weight bearing AP and lateral views that evaluated the position and fixation status of the components. Range of motion of the knees was measured at each follow-up. KSS score was measured preoperatively and at 3 months follow-up.

\section{Statistical analysis}

Statistical analysis was performed using Student's t-test. Correlation analysis was performed for $160^{\circ}<\mathrm{TFM}<195^{\circ}$ vs. $160^{\circ} \geq \mathrm{TFM} \geq 195^{\circ}$. Values of $\mathrm{p}<0.05$ were considered statistically significant. Statistical analyses were performed using PyQRS 4 software.

\section{Results}

\section{Preoperative data}

We divided the patients into two groups, one group of 60 patients with TFM between $160^{\circ}$ degrees and $195^{\circ}$ and the other group of 27 patients with TFM less than $160^{\circ}$ and more than $195^{\circ}$. There was no statistical difference between the two groups regarding age, sex, diagnosis (Table 1). The purpose of this study was to determine short-term impact in using the mini-subvastus approach in patients undergoing TKA with $160^{\circ}<\mathrm{TFM}<195^{\circ}$ compared to patients with $160^{\circ} \geq \mathrm{TFM} \geq 195^{\circ}$. No patients were lost to follow-up; all of them were followed for 3 months.

Table 1. Patients' demographics

\begin{tabular}{llll}
\hline & $160<T F M<195$ & $160 \geq T F M \geq 195$ & $\mathrm{p}$ \\
\hline No. of knees & 60 & 27 & $<0,05$ \\
No. of patients & 58 & 26 & $<0.05$ \\
Age (years)* & $67.9(54-82)$ & $68.3(56-80)$ & $<0.05$ \\
Gender (male/ & $19 / 31$ & $5 / 22$ & \\
female) & 93.3 & 92.4 & \\
Diagnosis (\% OA) & $60 / 0$ & $24 / 3$ & \\
Unconstrained/ & $89.3 \pm 10.2$ & $79.5 \pm 7.8$ & \\
Constrained & $50.5 \pm 9.5$ & $44.8 \pm 6.9$ & \\
Preoperative ROM & $184.1 \pm 3.2$ & $189.6 \pm 15.9$ & \\
Preoperative KSS & & & \\
Preoperative TFM & & & \\
\hline
\end{tabular}

*Values are given as mean $\pm S D ; O A$ - osteoarthritis

\section{Operative results}

Patients with TFM less than $160^{\circ}$ and more than $195^{\circ}$ experienced a longer operative time, with a mean of $127 \pm 8$ min, compared to patients with TFM between $160^{\circ}$ and $195^{\circ}$, with a mean of $104 \pm 9 \mathrm{~min}$. Incision length with the knee in extension was similar between the two groups with a mean value of $8.8 \mathrm{~cm}$ for $160^{\circ}<\mathrm{TFM}<195^{\circ}$ group and $9.5 \mathrm{~cm}$ for $160^{\circ} \geq \mathrm{TFM} \geq 195^{\circ}$ group (Table 2 ).

Table 2. Operative results

\begin{tabular}{llll} 
& $160<$ TFM $<195$ & $160 \geq$ TFM $\geq 195$ & $P$ \\
Operative time $(\mathrm{min})$ & $104 \pm 9$ & $127 \pm 8$ & \\
Blood loss $(\mathrm{ml}))^{*}$ & $535 \pm 187$ & $585 \pm 235$ & $>0.05$ \\
Incision length $(\mathrm{cm})$ & $8.8 \pm 1.4$ & $9.5 \pm 1.9$ & $0.05<p<0.1$ \\
\hline
\end{tabular}

*Blood loss measured intraoperatively plus that from suction drain 1 day postoperative; Values are given as mean $\pm S D$.

The total blood loss was $50.9 \mathrm{ml}$ greater for the second group, this being attributable to the magnitude of soft tissue release and to the differences between the implants used, mentioning that 3 patients from $160^{\circ} \geq$ $\mathrm{TFM} \geq 195^{\circ}$ group, who had undergone TKA using constrained non-hinged and hinged prosthesis (Fig. 1). There were no intraoperative complications for either of the groups. 


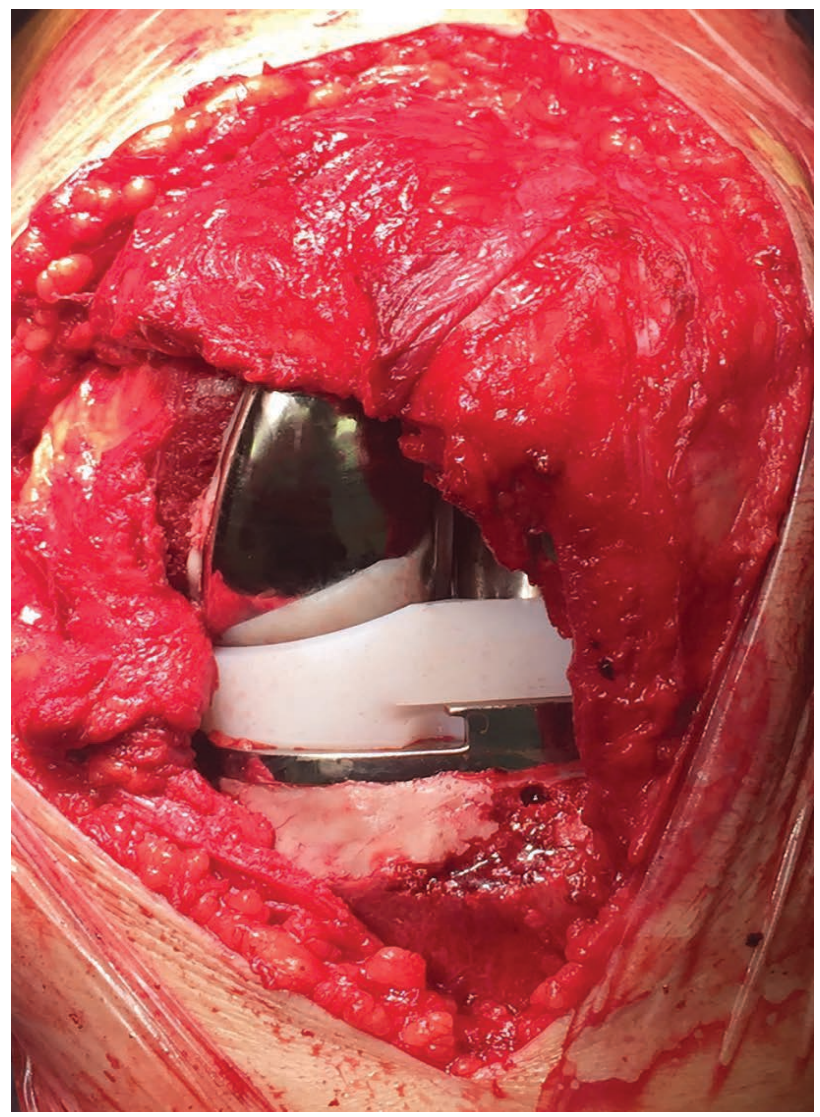

Fig. 1 MIS TKA of a knee performed through a minisubvastus approach with the final components inserted. $94 \times 147 \mathrm{~mm}(300 \times 300 \mathrm{DPI})$

\section{Postoperative clinical and functional results}

No significant differences in the knee score (KSS) were seen postoperatively at 3 months follow-up between the groups. Although the preoperative scores in $160^{\circ} \geq \mathrm{TFM} \geq 195^{\circ}$ group were inferior to the other group, the scores improved at 3 months postoperatively, with a higher rate of improvement. Postoperative improvement of the ROM was similar in both groups at three months follow-up (Table 3). There was no significant difference to the time to actively make a flexion of $90^{\circ}$ in a seated position, the ability to ambulate, use stairs, independent with home exercise program.

\section{Radiographic outcome}

Mean values of each angle were compared between $160^{\circ}<\mathrm{TFM}<195^{\circ}$ and $160^{\circ} \geq \mathrm{TFM} \geq 195^{\circ}$ groups (Table 4). Postoperative TFM, coronal femoral component angle (a), coronal tibial component angle (b), sagittal femoral component angle (c), and (d) sagittal tibial component angle were measured. We also analyzed postoperative FCM angle, the InsallSalvati ratio, and the patellar tilt. No significant differences were seen in the two groups. In both groups, the implants were aligned within $\pm 3^{\circ}$ from the ideal TFM in more than $80 \%$, with a higher TFM angle in $160^{\circ} \geq \operatorname{TFM} \geq 195^{\circ}$ group $(p<0.01)$. The FCM angle was lower in the second group, with a mean value of $82^{\circ} \pm 1.94$ ( $p>0.1$ ). The coronal femoral component angle was between normal values in both groups ( $p>0.1$ ). The coronal tibial component angle had a slightly lower value in the second group, with a tendency of varus malposition $(0.01<p<0.05)$. Sagittal femoral component angle had similar values in both groups $(0.01<p<0.05)$. The tibial slope or the sagittal tibial component angle measured postoperatively in both groups was within $\pm 2^{\circ}$ from the ideal of $83^{\circ}$ in $160^{\circ}<\mathrm{TFM}<195^{\circ}$ group and within $\pm 3^{\circ}$ from the ideal in $160^{\circ} \geq \mathrm{TFM} \geq 195^{\circ}$ group $(0.01<\mathrm{p}<0.05)$. The postoperative Insall-Salvati ratio was between normal values in both groups. The $160^{\circ} \geq \mathrm{TFM} \geq 195^{\circ}$ group displayed lower patellar tilt angle compared to the $160^{\circ}<\mathrm{TFM}<195^{\circ}$ group $(p<0.01)$.

Table 3. Clinic

\begin{tabular}{llll}
\hline & $160<$ TFM $<195$ & $160 \geq T F M \geq 195$ & $\mathrm{P}$ \\
& 3 M.Postoperative & 3 M.Postoperative & \\
Postoperative KSS & $87.7 \pm 5.5$ & $83.4 \pm 7.6$ & $<0.05$ \\
ROM (flexion) & $110.8 \pm 8.7$ & $101.1 \pm 8.5$ & $<0.05$ \\
\hline
\end{tabular}

Values are given as mean $\pm S D$.

Table 4. Radiographic analysis

\begin{tabular}{llll} 
& $160<T F M<195$ & $160 \geq T F M \geq 195$ & $\mathrm{P}$ \\
Preoperative TFM & $184.1 \pm 3.2$ & $189.6 \pm 15.9$ & $<0.05$ \\
Postoperative TFM & $180.6 \pm 1.8$ & $182.4 \pm 2$ & $<0.05$ \\
FCM & $89.3 \pm 1.7$ & $88.8 \pm 1.9$ & $<0.48$ \\
a & $95.9 \pm 1.5$ & $95.7 \pm 1.1$ & $<0.66$ \\
b & $89.2 \pm 1.6$ & $87.6 \pm 1.7$ & $<0.02$ \\
c & $2.6 \pm 2.2$ & $2.6 \pm 1.3$ & $<0.02$ \\
d & $83.9 \pm 1.4$ & $84.9 \pm 1.8$ & $<0.03$ \\
\hline
\end{tabular}

Values are given as mean $\pm S D$. 


\section{Discussion}

Minimally invasive surgery (MIS) in total knee arthroplasty (TKA) is a safe technique that spares the quadriceps tendon, decreases patient morbidity, and increases return to function, but how much deformity can be accepted for MIS TKA remains unknown. The benefits of mini-subvastus approach are widely accepted with similar results compared to classic approaches used in TKA. The good postoperative results might come from restrictive inclusion criteria not from the approach itself. This study focused on the effects of preoperative severity of varus more than $15^{\circ}$ and valgus more than $20^{\circ}$ on postoperative outcomes with mini-subvastus approach for TKA, using the radiographic analyses of implant positioning. Analyzing the postoperative radiographic parameters of implants positioning, the group with TFM lower than $160^{\circ}$ and higher than $195^{\circ}$ had satisfactory results compared to the group in which TFM was between $160^{\circ}$ and $195^{\circ}$. Differences existed in some parameters between the two groups. The group with TFM lower than $160^{\circ}$ and higher than $195^{\circ}$ had poorer radiographic results regarding postoperative TFM and FCM compared to the other group. One of the main intraoperative difficulties was to gain accurate coronal alignment, on radiographic analysis of the coronal tibial component angle, which was lower in $160^{\circ} \geq \mathrm{TFM} \geq 195^{\circ}$ group, with a slight tendency of varus malalignment. The coronal alignment that deviates more than $3^{\circ}$ from the ideal increases the risk of early defixation/ decimentation of the prosthesis $[20,21]$. Twenty-seven knees had TFM angle less than $160^{\circ}$ and more than $195^{\circ}$, from this group, 24 of them were performed using posterior cruciatesubstituting designs and 3 using constrained designs ( 1 hinged and 2 non-hinged) (Fig. 2). The operative results and the postoperative radiographic measurements of components alignment were the same, apart from the prosthesis design.
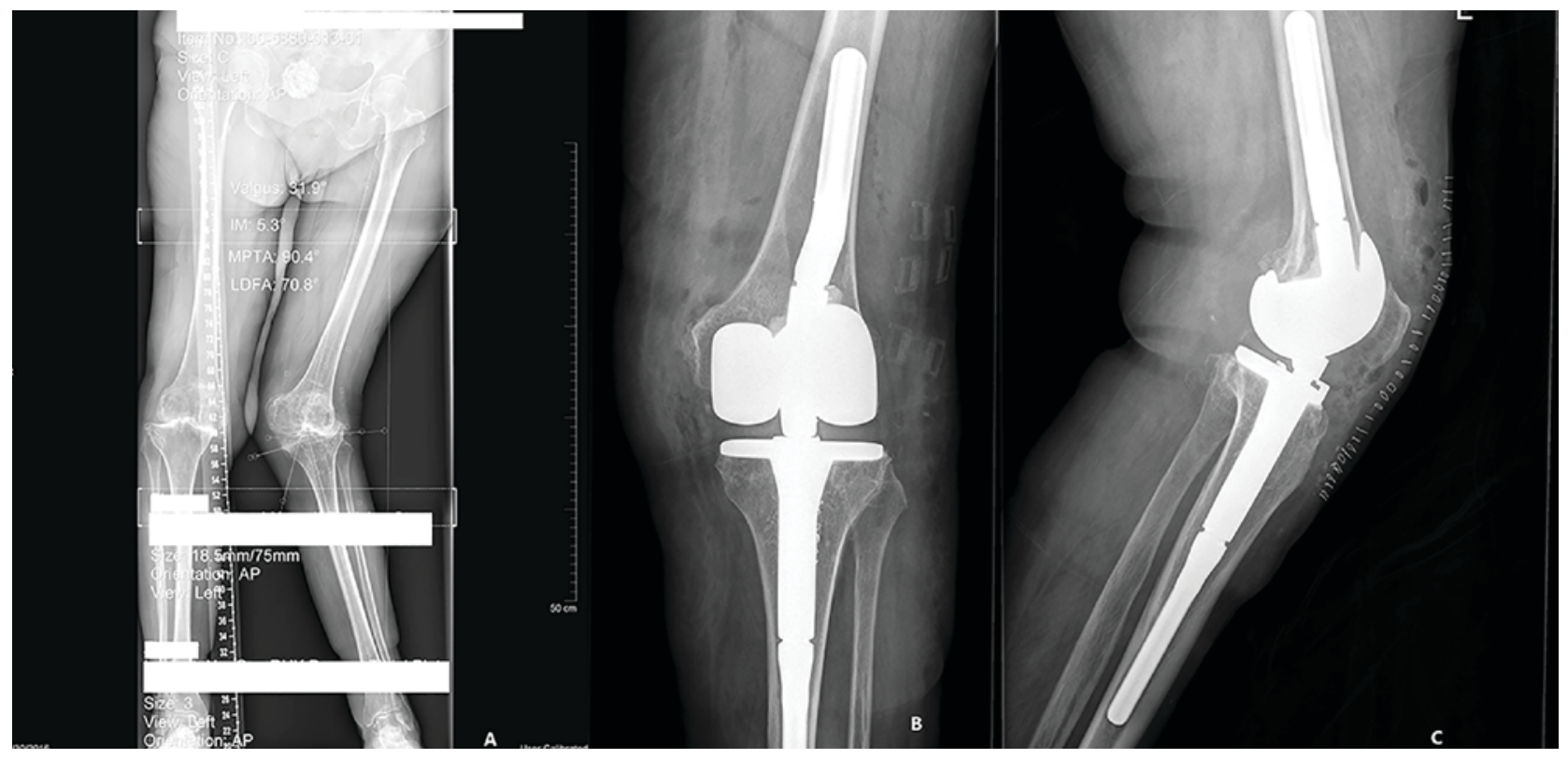

Fig. 2 Preoperative planning and postoperative radiographic evaluation. A: Preoperative planning to a genu valgum knee of 31.90, with knee instability. B, C: The patient underwent TKA with mini-subvastus approach using a constrained hinged prosthesis design. $215 \times 109 \mathrm{~mm}(300 \times 300 \mathrm{DPI})$

The rate of complications associated with mini-subvastus technique like femoral notching, problems in cementation, deep infection, and others were the same in both groups. One limitation of our study was the use of 2 dimensional (2D) analysis for implants positioning. Moreover, the 2D analysis of the rotational alignments of the femoral and 
tibial component were not evaluated, also the radiographic measurements of coronal and sagittal planes were not accurate because the $2 \mathrm{D}$ imaging did not ensure accurate anteroposterior and lateral views. Other limitations of this study are the relative small number of patients and the short period of follow-up. Further studies should be conducted on more patients on a longer period to confirm that long-term results are the same for $160^{\circ}<\mathrm{TFM}<195^{\circ}$ and for $160^{\circ} \geq \mathrm{TFM} \geq 195^{\circ}$.

\section{Conclusion}

The clinical and radiographic results of this study indicated that the mini-subvastus approach could be used safely in total knee arthroplasty with TFM angle less than $160^{\circ}$ and more than $195^{\circ}$ with similar results with TFM angle between $160^{\circ}$ and $195^{\circ}$. The minisubvastus approach can also be used in primary TKA using constrained non-hinged and hinged designs with promising results. Preoperative TFM less than $160^{\circ}$ and more than $195^{\circ}$ increases the risk of components malposition when using the mini-subvastus approach, with postoperative TFM more than $\pm 3^{\circ}$ from normal.

\section{References}

1. Tria Jr AJ. Advancements in minimally invasive total knee arthroplasty. Orthopaedics. 2003; 26(Suppl):859.

2. Bounutti PM, Mont MA, McMahon M et al. Minimally invasive total knee arthroplasty. J Bone Joint Surg. 2004; 86-A:26.

3. Laskin RS, Beksac B, Phongjunakorn A et al. Minimally invasive total knee replacement through a mini-midvastus incision: an outcome study. Clin Orthop. 2004; 428:74.

4. Aglietti P, Baldini A, Giron F et al. Minimally Invasive Total Knee Arthroplasty: Is it for Everybody?. HSSJ. 2006; 2:22-26.

5. Scuderi GR, Tenholder M, Capeci C et al. Surgical approaches in mini-incision total knee arthroplasty. Clin Orthop. 2004; 428:61.

6. Scuderi GR, Tria Jr AJ. Minimal incision total knee arthroplasty. In: Scuderi GR, Tria AJ. MIS of the Hip and the Knee: a Clinical Perspective, 2004, New York, Springer Verlag, 175.

7. Schroer WC, Diesfeld PJ, Reedy ME et al. Mini-Subvastus
Approach for Total Knee Arthroplasty. The Journal of Arthroplasty. 2008; 23:1.

8. Goble EM, Justin DF. Minimally invasive total knee replacement: principles and technique. Orthop Clin North Am. 2004; 35:235.

9. Tria Jr A. Minimally invasive total knee arthroplasty: the importance of instrumentation. Orthop Clin North Am. 2004; 35:227.

10. Choi YJ, Tanavalee A, Chan APH et al. Minimally invasive surgery for total knee arthroplasty. In: Scuderi GR, Tria AJ. MIS of the Hip and the Knee: a Clinical Perspective, 2004, New York, Springer-Verlag, 160.

11. Hoffmann AA, Plaster RL, Murdock LE et al. Subvastus (southern) approach for primary total knee arthroplasty. Clin Orthop. 1991; 269:70.

12. Wegrzyn J, Parratte S, Coleman-Wood K et al. The John Insall Award No Benefit of Minimally Invasive TKA on Gait and Strength Outcomes A Randomized Controlled Trial. Clin Orthop Relat Res. 2013; 471:46-55.

13. Haas SB, Cook S, Beksac B et al. Minimally invasive total knee replacement through a mini midvastus approach. Clin Orthop. 2004; 428:68.

14. Scuderi GR, Tria AJ Minimally Invasive Total Knee Artroplasty. In: Scott WN, Clarke HD. Insall \& Scott Surgery of the Knee. 2006, Philadelphia, Churchill Livingstone, Elsevier, 1631.

15. Khanna A, Gougoulias N, Longo UG et al. Minimally invasive total knee arthroplasty: a systematic review. Orthop Clin North Am. 2009; 40:479-489.

16. Moreland JR. Mechanisms of failure in total knee arthroplasty. Clin Orthop Relat Res. 1988; 226:49-64.

17. Matsumoto T, Muramatsu H, Kawakami Y et al. Soft tissue balance measurement in minimal incision surgery compared to conventional total knee arthroplasty. Knee Surg Sports Traumatol Arthrosc. 2011; 19:880-886.

18. Ewald FC. The knee society total knee arthroplasty roentgenographic evaluation and scoring system. Clin Orthop Relat Res. 1989; 248:9-12.

19. Gomes LSM, Bechtold JE, Gustilo RB et al. Patellar prosthesis positioning in total knee arthroplasty. Clin Orthop. 1988; 236:72.

20. Ritter MA, Faris PM, Keating EM et al. Postoperative alignment of total knee replacement. Its effect on survival. Clin Orthop. 1994; 299:153.

21. Rand JA, Coventry MB. Ten-year evaluation of geometric total knee arthroplasty. Clin Orthop. 1988; 232:168-73. 